\title{
Absorber Device
}

National Cancer Institute

\section{Source}

National Cancer Institute. Absorber Device. NCI Thesaurus. Code C50372.

A device or material designed to take in or attenuate a force or substance. 